\title{
OPEN Controlling long-term SARS-CoV-2 infections can slow viral evolution and reduce the risk of treatment failure
}

\author{
Debra Van Egeren ${ }^{1,2,3}$, Alexander Novokhodko ${ }^{4}$, Madison Stoddard ${ }^{5}$, Uyen Tran $^{5}$, \\ Bruce Zetter ${ }^{1,3}$, Michael S. Rogers ${ }^{1,3}$, Diane Joseph-McCarthy ${ }^{6}$ \& Arijit Chakravarty ${ }^{5 凶}$
}

The rapid emergence and expansion of novel SARS-CoV-2 variants threatens our ability to achieve herd immunity for COVID-19. These novel SARS-CoV-2 variants often harbor multiple point mutations, conferring one or more evolutionarily advantageous traits, such as increased transmissibility, immune evasion and longer infection duration. In a number of cases, variant emergence has been linked to long-term infections in individuals who were either immunocompromised or treated with convalescent plasma. In this paper, we used a stochastic evolutionary modeling framework to explore the emergence of fitter variants of SARS-CoV-2 during long-term infections. We found that increased viral load and infection duration favor emergence of such variants. While the overall probability of emergence and subsequent transmission from any given infection is low, on a population level these events occur fairly frequently. Targeting these low-probability stochastic events that lead to the establishment of novel advantageous viral variants might allow us to slow the rate at which they emerge in the patient population, and prevent them from spreading deterministically due to natural selection. Our work thus suggests practical ways to achieve control of long-term SARS-CoV-2 infections, which will be critical for slowing the rate of viral evolution.

The widespread deployment of biomedical interventions against SARS-CoV-2 has highlighted viral evolution as a significant potential risk in bringing the ongoing pandemic to an end. SARS-CoV-2 has a high mutational rate, similar to other RNA viruses, and a high mutational tolerance in key proteins, such as Spike, the molecular target for many biomedical interventions against the disease ${ }^{1-3}$.

The emergence and expansion of novel SARS-CoV-2 variants over the past few months threatens to undermine the promise of a return to normalcy as a result of the newly deployed vaccines. In fact, many of these new variants are more capable of infecting cells, spreading between hosts, and/or evading natural immunity or therapeutics ${ }^{4-6}$. On a population level, natural selection has acted to rapidly and deterministically increase the frequency of these more-fit SARS-CoV-2 variants, leading them to dominate the local viral population after emergence (Table S1). The emergence of fitter variants has also led in a number of cases to more severe disease outbreaks, and poses the threat of eventual reductions in vaccine efficacy ${ }^{4,5,7,8}$.

In addition to acting at the population level, natural selection has been shown to select for advantageous viral variants that are generated within individual patients infected with SARS-CoV-2. Longitudinal sequencing of SARS-CoV-2 from individual patients has revealed selection for multiple antibody-evading mutations, particularly in patients with long-term infections treated with convalescent plasma ${ }^{9}$. Additionally, studies have shown that individuals with impaired immune function can shed high levels of virus for weeks, creating an environment where SARS-CoV-2 is exposed to prolonged selection pressures favoring variants that can escape the immune response and/or are resistant to treatment ${ }^{10-14}$.

Understanding the factors driving the evolutionary process for SARS-CoV-2 could potentially allow us to design and use biomedical interventions in a way that hinders viral evolution, giving us the upper hand in managing the pandemic. In particular, there are several steps in the process of generating new advantageous variants that are stochastic, occurring largely by random chance.

${ }^{1}$ Harvard Medical School, Boston, MA, USA. ${ }^{2}$ Dana-Farber Cancer Institute, Boston, MA, USA. ${ }^{3}$ Boston Children's Hospital, Boston, MA, USA. ${ }^{4}$ University of Washington, Seattle, WA, USA. ${ }^{5}$ Fractal Therapeutics, Cambridge, MA, USA. ${ }^{6}$ Boston University, Boston, MA, USA. ${ }^{\boxplus}$ email: arijit@fractaltx.com 
First, sufficient genetic diversity must be created within infected individuals through stochastic events. SARSCoV-2 mutations are initially generated by random errors in viral replication within individuals with COVID19. Deep sequencing studies have revealed that the SARS-CoV-2 viral population exists within the host as a quasispecies $^{15-18}$, a population structure with a large number of related sequences arising from de novo mutations that occur during the course of infection. Quasispecies genetic diversity has been shown to vary over time $e^{16,19}$, and deep sequencing studies have demonstrated a role for genetic drift ${ }^{20}$ and intrahost transmission bottlenecks ${ }^{15}$ as the virus moves from one region of the body to another. While genetic diversity provides opportunities for advantageous mutations to arise and expand due to natural selection within individuals infected with SARS$\mathrm{CoV}-2$, genetic drift may provide a barrier to the deterministic expansion of advantageous viral mutations at lower population sizes.

Next, viral variants generated within a COVID-19 patient are transmitted to new hosts. During this process, further stochasticity is introduced by the low numbers of viral particles required to start an infection in a new host, which creates a narrow transmission bottleneck ${ }^{21,22}$. Consistent with this bottleneck, sequencing studies have provided conflicting results with respect to the ability of intrahost variants to transmit and establish new SARS-CoV-2 lineages that have the potential to transmit widely. While a number of studies have shown this to be the case $\mathrm{e}^{21-24}$, other studies have not been able to demonstrate transmission of viral lineages derived from intrahost evolution ${ }^{11,25,26}$. The stochasticity in inter-host transmission of specific viral lineages is compounded by the overdispersed nature of SARS-CoV-2 spread, where most onward transmission originates from a small number of individuals. Recent work also suggests an important role for stochastic extinction of variants, as at least five new infections are required in order for a newly emergent variant to establish itself in the population ${ }^{27}$.

These stochastic factors in the evolution of SARS-CoV-2 represent a potential weakness that can be exploited in the design of intervention strategies to slow viral evolution. To the extent that the stochastic contribution of drift can be increased, the deterministic contribution of natural selection to the improvement of viral fitness can be weakened. In this study, we have used evolutionary dynamics to better understand the process by which fitter SARS-CoV-2 variants arise during infections and to identify practical means by which viral evolution can be slowed.

\section{Results}

Single mutants that are fitter within patients are more likely to be transmitted to new hosts. To investigate mutation and selection dynamics of SARS-CoV-2 within hosts, we simulated stochastic viral evolution using a modified Wright-Fisher model (Fig. 1A, Methods). During each 12-h replication cycle, virions from the previous generation are randomly selected with probability proportional to their fitnesses to replicate and produce a burst of $N_{\mathrm{b}}$ new viral particles in the next generation. Each replicating virion has a constant probability of generating a new single point mutation that is passed to all of its $N_{\mathrm{b}}$ progeny. The total number of virions present in each generation is given by estimates from previously-published sputum RT-PCR measurements ${ }^{28}$ (Fig. 1B, Methods). Unless stated, the parameter values for the simulations are those given in Table 1.

Over the course of a typical-length COVID-19 infection (23 days), our simulations show that viral variants with point mutations that increase the replication probability by $20-50 \%$ (selection coefficients of $0.2-0.5$ ) expand significantly more than variants with neutral or weakly deleterious fitness effects (Fig. 1C). This expansion of fitter variants increases the probability that at least one viral particle with a specific beneficial mutation will be transmitted to a new host (Fig. 1D, Methods). Individuals are more likely to pass on beneficial variants if transmission occurs later in the infection, since the frequency of these variants increases over time due to selection. Assuming these variants are also able to spread through the population with a moderate transmission advantage, new lineages with advantageous single mutations are rapidly created at the population level (Fig. 1E). These results suggest that selection for beneficial single point mutations within COVID-19 patients increases the rate at which fitter SARS-CoV-2 lineages establish at a population level.

Longer infection duration and higher viral load increase probability of transmitting fitter variants. Viral load dynamics vary significantly between COVID-19 patients ${ }^{28}$. To assess the evolutionary consequences of this variation, we simulated viral replication and transmission of variants for patients with different viral load kinetics (Fig. 2). Patients with longer periods of peak viral load were able to transmit fitter SARSCoV-2 variants more efficiently (Fig. 2A). On the other hand, decreasing viral load decreased the probability that fitter variants generated within a patient would be transmitted (Fig. 2B). Both of these observations indicate that the strength of selection increases as the number of replicating viruses over the course of an infection increases.

Beneficial two-mutation combinations are readily generated within patients with long-term SARS-CoV-2 infections. Many of the reported new SARS-CoV-2 variants are defined by more than one point mutation. One of these variants arose during long-term SARS-CoV-2 infection within an immunocompromised individual and included a mutation that conferred resistance to neutralizing antibodies but reduced infectivity, which was offset by a mutation that increased infectivity. These observations suggest that highly-fit mutation combinations that require transit through a deleterious intermediate state (i.e., crossing a fitness valley) may be generated within COVID-19 patients with longer infection durations.

To investigate the rate at which these variants are generated within hosts, we modeled a multistep mutation process where beneficial mutation combinations are created from deleterious intermediates that only have some of the mutations found in the beneficial combination (Fig. 3A). Beneficial two-mutation combinations exist at very low frequencies over the timescale of a typical-length SARS-CoV-2 infection but increase in frequency within hosts that have prolonged infections (Fig. 3B). This increase in variant frequency that occurs within 
A
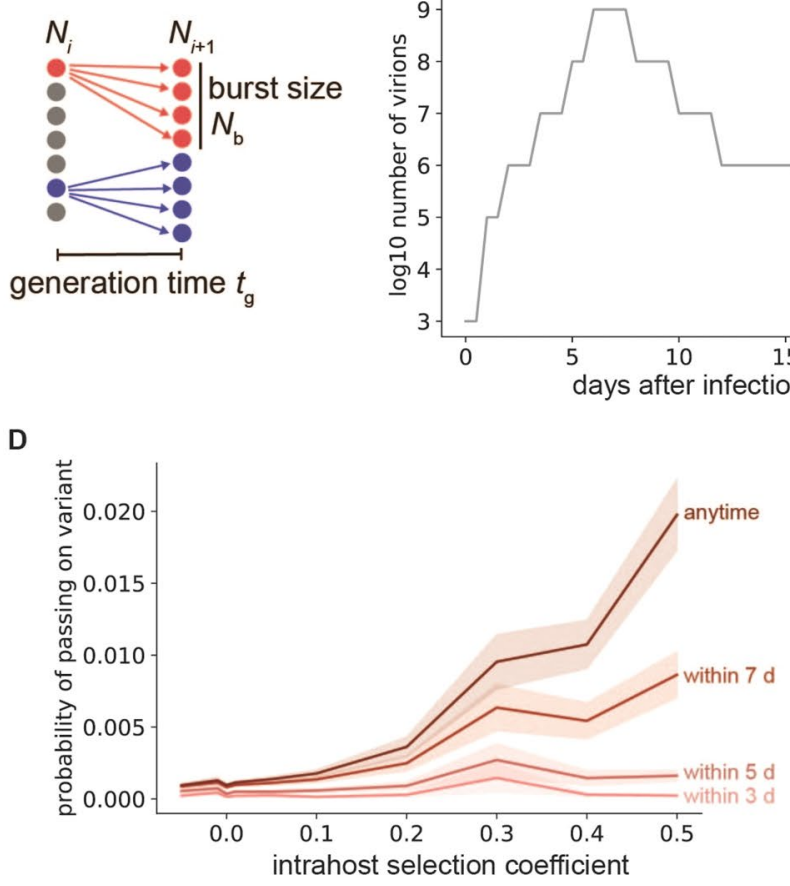

C

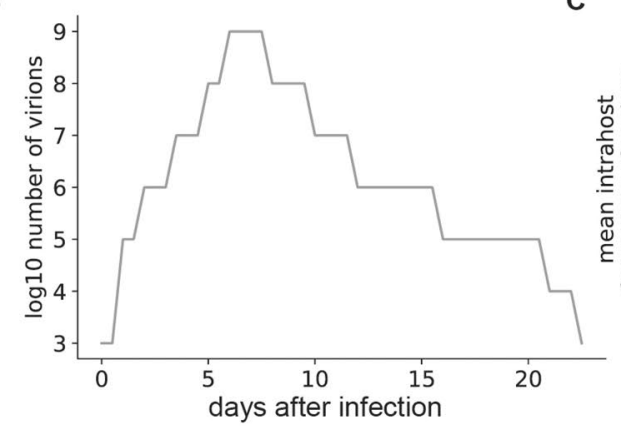

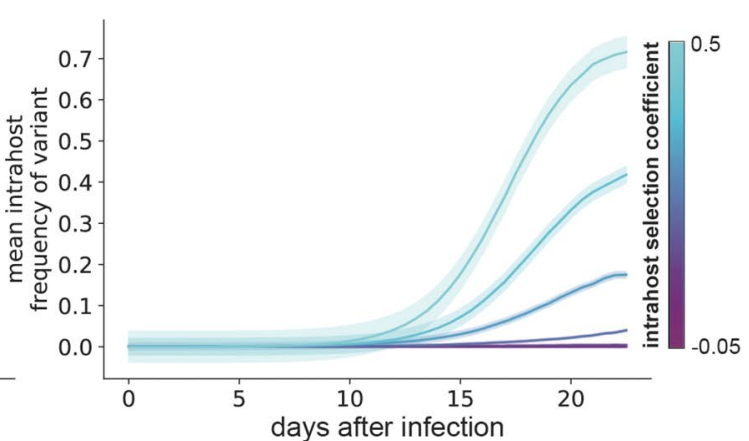

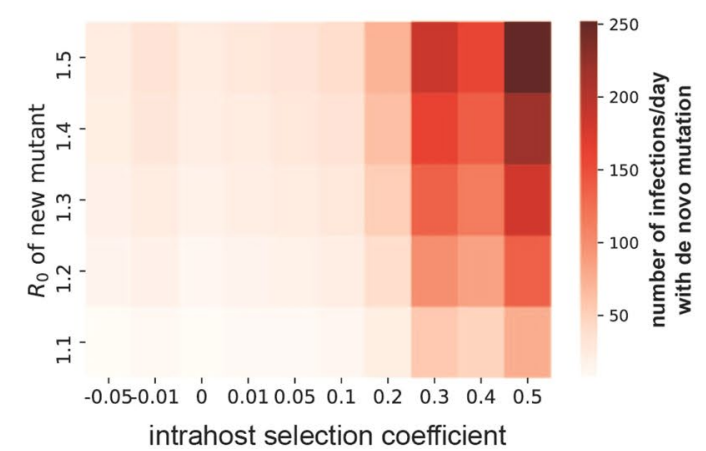

Figure 1. Selection within individuals with COVID-19 leads to selection of more fit viral variants. (A) Schematic of viral replication model used to simulate SARS-CoV-2 evolutionary dynamics. Colors denote the descendants created in generation $N_{i+1}$ by the replication burst of each individual ancestor in generation $N_{i}$. (B) Sputum viral load curve for a typical COVID-19 infection. The x-axis represents the time starting from the initial transmission event that caused the infection. (C) Mean frequency of variants with point mutations within individuals with COVID-19 for different mutation fitness effects (colors). (D) Probability of a specific single mutation to be present in at least one virion transmitted if transmission occurs within the first 3-7 days of infection (lighter red curves) or anytime during infection (darker red). For (C) and (D), shaded areas represent \pm SEM, $n=1000$ simulations per condition. (E) Number of total new single mutant infections generated per day that establish a surviving variant lineage, assuming all infections are of standard length with viral load profile given in (B) and that transmission occurs within the first 7 days of infection. Unless otherwise specified, simulation parameter values are those given in Table 1.

\begin{tabular}{|c|c|c|c|}
\hline Name & Description & Value & Source \\
\hline$t_{\mathrm{g}}$ & SARS-CoV-2 replication cycle length & $12 \mathrm{~h}$ & 57 \\
\hline$N_{\mathrm{b}}$ & Viral burst size-number of virions produced by each infected cell & 1000 & 57 \\
\hline$\mu$ & Within-host SARS-CoV-2 mutation rate (per replication cycle, per site) & $10^{-5}$ & 57 \\
\hline$N_{\text {trans }}$ & Number of virions transmitted to new host & 10 & 22 \\
\hline$R_{0}$ & $\begin{array}{l}\text { Reproductive number-average number of new hosts infected by a single host infected } \\
\text { with the mutant virus }\end{array}$ & 1.5 & Similar to the transmission advantage measured for B.1.1. $7^{5}$ \\
\hline$\sigma$ & Number of new infected hosts (per day) & 50,000 & Similar to recently reported numbers for the US \\
\hline$L_{\mathrm{LT}}$ & Mean ( \pm standard deviation) of infection length in long-term SARS-CoV-2 shedders & 93.75 days \pm 11.48 & 56 \\
\hline
\end{tabular}

Table 1. Parameter values used for intrahost SARS-CoV-2 evolutionary dynamics simulations.

hosts due to selection corresponds to an increase in the probability the beneficial two-mutation variant will be transmitted (Fig. 3C). Therefore, patients with longer SARS-CoV-2 infections are more likely to transmit a variant with multiple mutations. On a population level, these individuals who produce and shed virus for long periods ( $>30$ days after symptom onset) increase the production rate of new variants with multiple mutations. Increasing the proportion of COVID-19 patients with prolonged SARS-CoV-2 shedding increases the rate at which new, fitter variants with two mutations are produced (Fig. 3D).

SARS-CoV-2 evolution can be impeded by targeting stochastic events required for the emergence of new variants. Several steps are required for a new SARS-CoV-2 variant to be generated and established in the population (Fig. 4A), and each of these steps represents a potential choke point for viral evolution that can be exploited in the design of interventions. First, a new variant must be generated through mutation and expand within a host. The efficiency of generating and selecting advantageous variants within COVID-19 

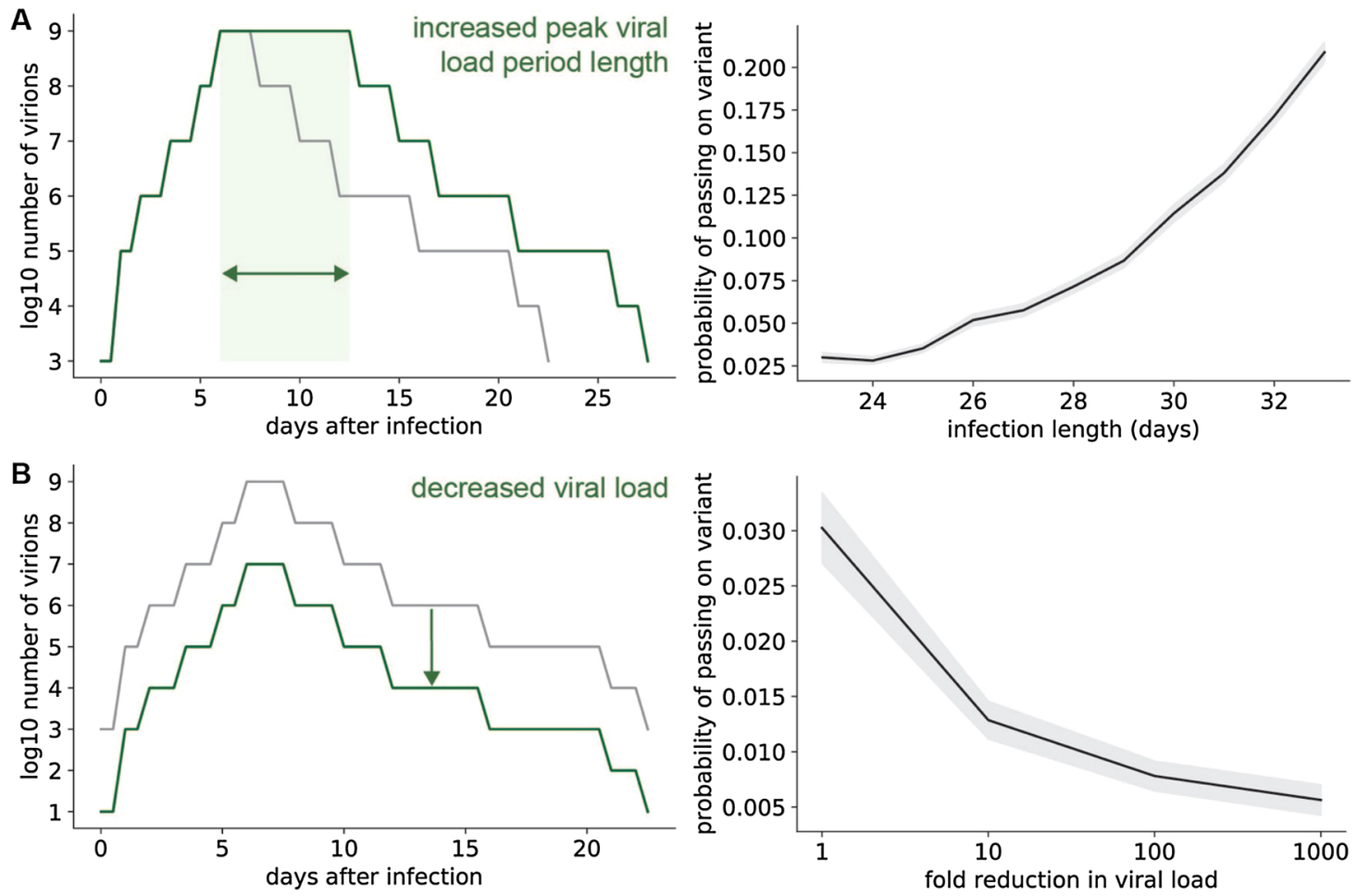

Figure 2. Within-host selection is stronger for infections that last longer or have higher viral loads. (A) (Left panel) example of increased peak viral load period length. (Right) Probability that at least one transmitted virion has a specific advantageous single mutation, for different overall lengths of infection. Infection lengths were adjusted by increasing the length of the peak viral load period (left panel schematic). (B) (Left panel) example of decreased viral load. (Right) Probability that at least one transmitted virion has a specific single mutation, for different viral loads. Viral loads were adjusted by reducing the viral load by a constant factor over the entire course of infection (left panel schematic). For right panels, shaded areas represent $\pm S E M, n=1000$ simulations per condition, with a selection coefficient of 0.2 for the single mutant. Unless otherwise specified, simulation parameter values are those given in Table 1.

patients can be reduced by biomedical interventions that reduce viral load within patients or reduce the frequency of long-term infections which lead to viral transmission (Fig. 4B). Variants then must be transmitted to additional hosts to establish within the population. Reducing the number of viral particles transmitted to new hosts (for example, through mask wearing or vaccination) will also slow the rate of emergence of advantageous variants (Fig. 4B). Finally, interventions specifically aimed at reducing the transmissibility of the new variant would also reduce their probability of spreading widely (Fig. 4B). This could be achieved by deliberately deploying a patchwork of vaccines and other prophylactics that target distinct epitopes, thereby increasing the diversity of biomedical interventions to provide a more challenging evasion landscape for the virus. Taken together, our simulation results suggest that there are several low-probability stochastic events that are important for SARS$\mathrm{CoV}-2$ variant emergence and that interventions targeting these events can slow SARS-CoV-2 evolution.

\section{Discussion}

The recent emergence of variants of SARS-CoV-2 with multiple mutations poses a direct threat to the viability of vaccine-based suppression strategies for the pandemic ${ }^{29}$. A number of phenotypic changes that are capable of providing a fitness advantage have been associated with newly emerged variants. These include increased transmissibility, lethality, and viral replication rate, all characteristics of the B.1.1.7 lineage ${ }^{5,30,31}$. Additionally, some variants have immune evading phenotypes, including increased reinfection potential (e.g., B.1.351) ${ }^{32}$, and reduced neutralization for monoclonal antibodies (e.g., B.1.1.7, P.2 and B.1.351) ${ }^{33,34}$, convalescent plasma (e.g., B.1.351, B.1.429) $)^{32,35}$, and vaccine-induced sera (e.g., B.1.351, B.1.429, B.1.1.7, B1.298, P.2, and P.1) ${ }^{36,37}$ or complete resistance to a vaccine (B.1.351) ${ }^{37}$ (see Supplementary Note and Supplementary Table S1 for further details). Some viral variants have also been associated with a longer duration of infection ${ }^{38}$, which is particularly concerning in light of our finding that longer infections increase the probability of transmitting fitter SARS-CoV-2 variants.

In this work, we investigated the impact of within-host SARS-CoV-2 mutation and selection on the emergence of new viral variants in the population. Using a stochastic computational model of viral replication and selection, we found that mutations that increased the rate of viral replication increased in frequency within hosts during the course of a typical SARS-CoV-2 infection. This expansion led to more frequent transmission of the new variant and faster emergence of the variant on a population level. The effect of selection within hosts is more pronounced for longer SARS-CoV-2 infections with higher viral load, which can lead to the generation of variants 
A

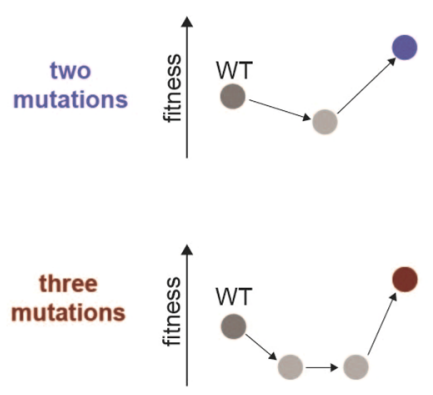

C

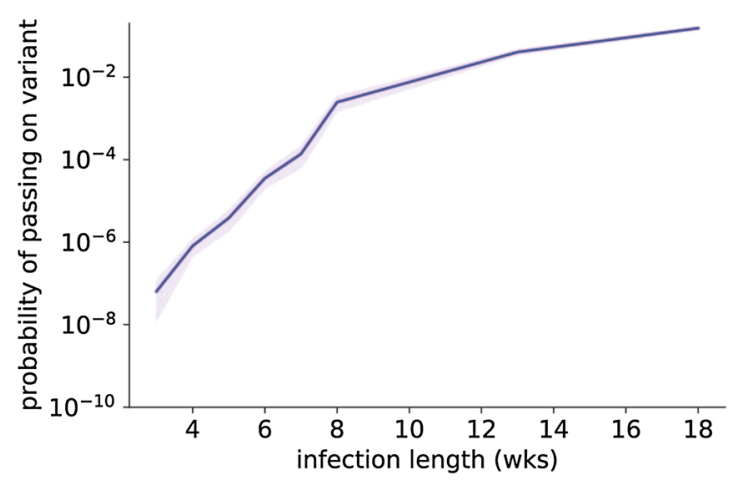

B

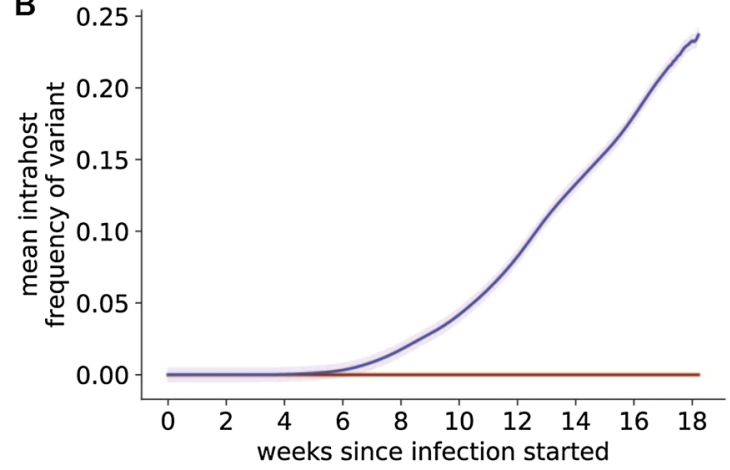

D

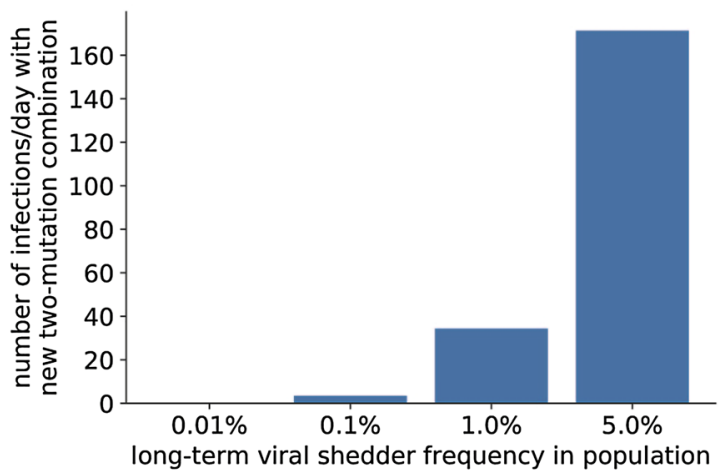

Figure 3. SARS-CoV-2 can acquire multiple mutations during infections with sustained viral replication. (A) Fitness valley crossing model for acquisition of multiple mutations. Intermediate states with fewer mutations (light grey) have lower fitness than the WT virus within individuals, while variants with a specific combination of two or three mutations (blue and red, respectively) have higher fitness. (B) Mean frequency of variants with a beneficial combination of two (blue) or three (red) mutations within individuals with long-term SARS-CoV-2 infection. (C) Probability of a beneficial combination of two mutations (blue line) to be present in at least one virion transmitted if transmission occurs anytime during infection. For (B) and (C), shaded areas represent \pm SEM, $\mathrm{n}=1000$ simulations per condition. (D) Number of de novo double mutant infections that establish a surviving lineage when some COVID-19 patients shed live virus for more than 30 days after developing symptoms. Deleterious intermediates had a fitness cost of 0.05 and beneficial mutation combinations had a selective advantage of 0.2 . Unless otherwise specified, simulation parameter values are those given in Table 1.

with multiple mutations within a single host during prolonged infection. While these general findings are in line with the intuition provided by evolutionary theory and viral quasispecies modeling 39 , our findings suggest that the expected frequency of these variant-generation events (using real-world parameter estimates) is likely to be high enough to represent a tangible public health threat. The risk to public health from long-term infections is not currently appreciated, as public-health authorities continue to use symptom-based strategies for testing and discontinuing transmission-based precautions ${ }^{40}$ even if the patient tests positive for SARS-CoV-2 RNA in a nasal swab ${ }^{41}$. Our work also points to a strategy for controlling viral evolution-the stochastic events required for variant generation represent a vulnerability from the virus' perspective which can be exploited in the design of potential biomedical interventions. Thus, our quantitative modeling points to real-world practical risks that need to be mitigated as the pandemic drags on, and points out a way in which this can be done.

Our modeling approach has several limitations and assumptions that could be further investigated. When modeling intrahost viral evolution, we assumed that patients were initially infected with only wild-type virus. We did not account for genetic variation in SARS-CoV-2 already present in the population due to neutral genetic drift, which is another important source of advantageous viral mutations ${ }^{3}$. Our model did not account for variation in some parts of the viral replication cycle, including replication cycle length and burst size, which may represent additional stochastic events that could influence viral evolution. We also only modeled point mutations, which does not account for other possible genetic alterations that affect viral fitness (indels or rearrangements). Our population-level transmission model also does not consider the effects of spatial or demographic structure on SARS-CoV-2 spread. Finally, we only investigated the case where mutations that increase viral fitness within hosts also increases transmissibility on a population level. This hypothesis is consistent with the observation that increased viral load is associated with increased transmissibility ${ }^{42}$.

This work, along with other recent findings on the stochastic nature of SARS-CoV-2 transmission ${ }^{27}$, suggests a number of real-world strategies that can be used to suppress the emergence of these fitter viral variants, thereby slowing the evolution of SARS-CoV-2. First, long-term SARS-CoV-2 infections (lasting longer than 30 days) should be treated as a serious public health concern, regardless of the presence of symptoms. Our work suggests 
A

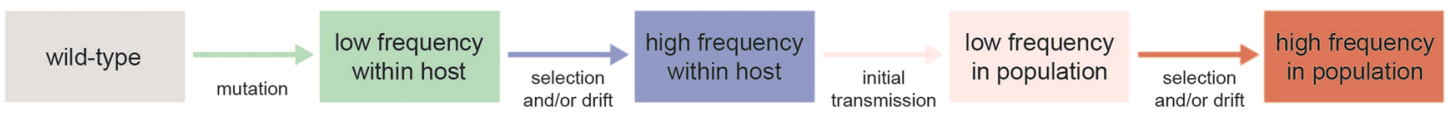

B

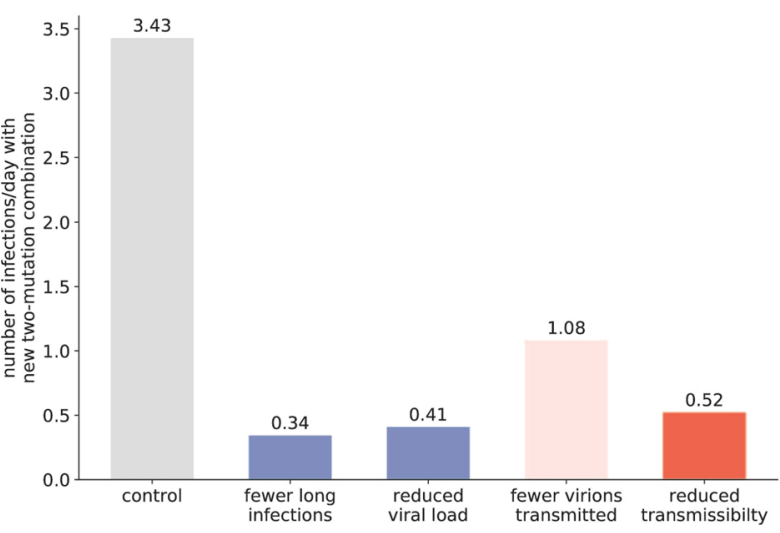

Figure 4. Interventions targeting stochastic events during infection or transmission reduce the efficiency of SARS-CoV-2 evolution. (A) Schematic showing events necessary for the generation and population-level establishment of a new SARS-CoV-2 variant. (B) Reduction in generation rate of new double mutant lineages that establish in the entire population caused by interventions targeting different events required to create a new surviving viral variant $(\mathrm{n}=1000$ simulations per condition). Each intervention reduces the stated parameter value by $90 \%$. Parameter value changes are relative to the control parameter set given in Table 1 , with $0.1 \%$ of new infections lasting $>30$ days after symptom onset. The "reduced transmissibility" condition refers to a $90 \%$ reduction in the transmission advantage of the variant over wild-type under steady-state transmission conditions, leading to a lower reproductive number $R_{0}$ of 1.05 .

that the low frequency of such long-term cases belies the threat that they pose to public health. In particular, patients with asymptomatic long-term infections still transmit ${ }^{10-13}$ and can potentially be efficient accelerators of viral evolution. Thus, patients testing positive for SARS-CoV-2 by RT-PCR after 30 days should be tested for viable virus, and if found to remain infectious, should isolate. Isolation measures for such long-term infections should be designed to minimize transmission in the interest of minimizing their disproportionate impact on the pace of viral evolution. This would represent a departure from current practice (the CDC, for example, does not require negative testing prior to ending isolation for mild and asymptomatic cases of COVID-1943). Second, treatments for long-term viral infections should be aimed at the suppression of viral load and should be initiated regardless of whether patients are symptomatic. Such treatment regimens should consist of multiple active agents that can suppress viral replication and that preferably target different viral proteins other than Spike to reduce the risk of generating viral mutants that are resistant to the treatment. This shift in medical practice may reduce the risk of intrahost evolution leading to emergence of immune-evading SARS-CoV-2 variants, as likely occurred in some documented cases with convalescent plasma treatment ${ }^{9}$. Finally, contact tracing of transmission events and genetic characterization of secondary infections resulting from long-term infections (including those from immunosuppressed patients) will be crucial.

We note that detecting long-term infections with SARS-CoV-2 represents a major logistical challenge in its own right-a widely deployable method for distinguishing long-term infections from spurious PCR results is an unmet need at present. While PCR positivity tracks closely with infectious viral particles at the beginning of infection, the presence of neutralized virus at later timepoints make PCR positivity difficult to interpret as a marker of infectiousness ${ }^{44}$. Although viral culture can be used to confirm the presence of replication-competent virus $^{45}$, logistical constraints (such as turnaround time and the requirement for Biosafety Level 3 containment) limit its clinical use ${ }^{46}$. Subgenomic RNA (sgRNA) has been proposed as a biomarker for actively replicating virus $^{47}$, but this remains controversial at present ${ }^{48}$. Therefore, there is an urgent need to develop a validated biomarker for viral replication in long-term infections. Such a biomarker will also enable us to better measure the frequency of transmissible long-term infections and differentiate them from long-term COVID-19 sequelae in patients without infectious virus.

Our work also suggests reasons for concern if intrahost evolution in long-term SARS-CoV-2 infections is not addressed directly. As intrahost evolution leads to faster generation of beneficial variants at a population level, new waves of transmission driven by fitter viral variants can be expected to arise as a result of untreated long-term infections. Our results also suggest another evolutionary incentive favoring increased infection duration, since long-term SARS-CoV-2 infections increase the rate at which the virus evolves. To the extent that this evolution is shaped by immune evasion (and immune evasion is expected to result in more severe infections), this study identifies an additional evolutionary route that is open to SARS-CoV-2, by which the virus can evolve to become progressively more lethal. Notably, while this potential risk conflicts with recent work that has conjectured that SARS-CoV-2 evolution may lead to progressive decreases in viral virulence ${ }^{49}$, it is consistent with available real-world evidence for this virus so far $^{30}$. The evolution of increased virulence over time has been observed for 
several other viral pandemics, including for HIV over the years 1984-2010 ${ }^{50}$, the second wave of the 1918-1919 Influenza pandemic ${ }^{51}$, and myxomatosis in rabbits in the $1970 \mathrm{~s}$ and $80 \mathrm{~s}^{52-54}$.

The deployment of multiple vaccines against SARS-CoV-2 in under a year is a remarkable triumph of modern medicine. These vaccines hold the promise of bringing the current pandemic to an end but are vulnerable to the rapid emergence and expansion of immune-evading viral variants. Understanding the mechanism of SARS-CoV-2 evolution allows us to design strategies that can tip the balance in this evolutionary arms race and ultimately allow us to control the spread of SARS-CoV-2.

\section{Methods}

Intrahost viral dynamics simulations. We used a modified Wright-Fisher evolutionary model to investigate viral mutation and selection dynamics within individuals with COVID-19. This model assumes that SARS-CoV-2 virions replicate in discrete generations of length $t_{\mathrm{g}}$ and that each replicating virion produces a fixed burst size of new virions $N_{\mathrm{b}}$. The total number of virions present at each generation was estimated from previously-published nasopharyngeal swab and sputum qRT-PCR measurements after diagnosis ${ }^{28}$. This estimated viral load curve over time (shown in Fig. 1B) was used for the simulations shown in Fig. 1. In Figs. 2, 3 and 4, we used the original viral load curve as a baseline and varied the length of the peak viral load period and/ or scaled the total number of virions in each generation (Fig. 2).

During each replication cycle, $N_{\mathrm{t}} / N_{\mathrm{b}}$ virions from the previous generation are chosen to reproduce, where $N_{\mathrm{t}}$ is the total number of virions in the current generation and $N_{\mathrm{b}}$ is the burst size. Each virion from the previous generation has a probability of reproducing and contributing $N_{\mathrm{b}}$ virions to the next generation that is proportional to its fitness. During each replication, there is a constant probability of generating a particular mutation that will be present in all $N_{\mathrm{b}}$ virions that are produced by that parent virion. Within each simulation condition, mutations were assumed to provide the same constant fitness benefit or penalty compared to the wild-type virion fitness, provided as the intrahost selection coefficient value in the figures and figure captions. Parameter values common to all simulations are given in Table 1, and additional parameter values specific to individual conditions are given in the figure captions. Each condition was simulated 1000 times.

Probability of variant transmission from a single infected host. The probability that a transmission event that includes at least one mutant virion occurs within a particular time window during infection was estimated by assuming new infections are initiated by $N_{\text {trans }}$ virions sampled uniformly at random from the transmission period which go on to establish a new infection. Therefore, the probability that at least one virion with a specific mutation is passed on is $1-(1-f)^{N_{\text {trans }}}$, where $f$ is the fraction of virions with the mutation that existed within the transmission window.

Population-level de novo generation rate of SARS-CoV-2 variants. A newly-generated variant present in a single patient will initially spread stochastically to new hosts, the success of which will determine whether the variant will survive in the population. We modeled the stochastic spread of a new variant within the population as a branching process in which infected individuals infect on average $R_{0}$ new hosts. More specifically, each infected individual transmits to $X$ new hosts, where $X$ is a random variable distributed as $\operatorname{Poisson}\left(R_{0}\right)$. Probability theory results for Poisson branching processes states that if a new variant has $R_{0}>1$, it will survive in the population with probability $\pi$, where $\pi$ is the smallest solution to $\pi=1-e^{-R_{0} \pi}$ in the interval $[0,1]^{55}$. This model assumes the population is well-mixed, without any population or geographic structure affecting COVID19 transmission.

We combined this estimated survival probability of an existing variant in the population with the results from the intrahost simulations described above to calculate the overall rate at which new surviving variants arise. The rate at which new surviving variants are generated from patients with infections of length $i$ is $\pi \sigma_{i} p_{i}$, where $p_{i}$ is the probability of a patient with infection length $i$ generating and transmitting the variant and $\sigma_{i}$ is the number of patients with infection length $i$ that are infected each day. The total rate at which new surviving variants are generated in the population is just the sum over all infection lengths, $\sum_{i} \pi \sigma_{i} p_{i}$. To estimate $\sigma_{i}$, we assumed that most infections were of the typical length and viral load kinetics shown in Fig. 1B. However, a small fraction $p_{\mathrm{LT}}$ of infections were assumed to be longer than 30 days. The distribution of infection lengths in these long-term patients was assumed to be normal, and mean and standard deviation were estimated from longitudinal oral swab RT-PCR data ${ }^{56}$. The rate $z_{\mathrm{LT}}$ at which new surviving variants are generated from long-term patients was estimated by simulating infections that are $1,2,3,4,5,10$, or 15 weeks longer than typical infections to estimate $p_{i}$ for each infection length and multiplying by the fraction of long-term infections within the length interval ending at $i$. That is, $z_{L T}=\sum_{i} \pi \sigma_{i} p_{i}$ for $i$ in $\{30,37,44,51,58,95,128\}$ days, where $\sigma_{i}=\mathrm{P}(X<i)-\sigma_{i-1}$ and $X$ is normally distributed according to the distribution of infection lengths. The total rate at which new surviving variants are generated in the entire population is therefore $z_{\mathrm{LT}}+z_{\mathrm{ST}}$, where $z_{\mathrm{ST}}$ is the rate at which standard length infections produce the variant.

Interventions that reduce the rate at which new variants are generated were modeled by reducing the fraction of long-term infections (reduced from $0.1 \%$ to $0.01 \%$ ), number of virions transmitted during each infection event (reduced 10 to 1), overall viral load for each infection (reduced by $90 \%$ from the baseline curve given in Fig. 1B), or the transmission rate of the variant in the simulations (reduced from 1.5 to 1.05 ).

\section{Code availability}

Scripts for running the viral dynamics simulations and plotting the results can be found at https://github.com/ dvanegeren/covid-intrahost-evo. 
Received: 5 May 2021; Accepted: 27 October 2021

Published online: 19 November 2021

\section{References}

1. Starr, T. N. et al. Deep mutational scanning of SARS-CoV-2 receptor binding domain reveals constraints on folding and ACE2 binding. Cell 182, 1295-1310 (2020).

2. Baum, A. et al. Antibody cocktail to SARS-CoV-2 spike protein prevents rapid mutational escape seen with individual antibodies. Science 369, 1014-1018 (2020).

3. Van Egeren, D. et al. Risk of rapid evolutionary escape from biomedical interventions targeting SARS-CoV-2 spike protein. PLOS ONE 16, e0250780 (2021).

4. Abdool Karim, S. S. \& de Oliveira, T. New SARS-CoV-2 variants-clinical, public health, and vaccine implications. N. Engl. J. Med. 6, 67 (2021).

5. Davies, N. G. et al. Estimated transmissibility and impact of SARS-CoV-2 lineage B.1.1.7 in England. Science https://doi.org/10. 1126/science.abg3055 (2021).

6. Tada, T. et al. Decreased neutralization of SARS-CoV-2 global variants by therapeutic anti-spike protein monoclonal antibodies. bioRxiv https://doi.org/10.1101/2021.02.18.431897 (2021).

7. Tegally, H. et al. Emergence and rapid spread of a new severe acute respiratory syndrome-related coronavirus 2 (SARS-CoV-2) lineage with multiple spike mutations in South Africa. medRxiv https://doi.org/10.1101/2020.12.21.20248640 (2020).

8. Sabino, E. C. et al. Resurgence of COVID-19 in Manaus, Brazil, despite high seroprevalence. The Lancet 397, $452-455$ (2021).

9. Kemp, S. A. et al. SARS-CoV-2 evolution during treatment of chronic infection. Nature https://doi.org/10.1038/s41586-021-03291-y (2021).

10. Choi, B. et al. Persistence and Evolution of SARS-CoV-2 in an Immunocompromised Host. N. Engl. J. Med. 383, 2291-2293 (2020).

11. Valesano, A. L. et al. Temporal dynamics of SARS-CoV-2 mutation accumulation within and across infected hosts. bioRxiv https:// doi.org/10.1101/2021.01.19.427330 (2021).

12. Baang, J. H. et al. Prolonged severe acute respiratory syndrome coronavirus 2 replication in an immunocompromised patient. J. Infect. Dis. 223, 23-27 (2021).

13. Truong, T. T. et al. Persistent SARS-CoV-2 infection and increasing viral variants in children and young adults with impaired humoral immunity. medRxiv https://doi.org/10.1101/2021.02.27.21252099 (2021).

14. Avanzato, V. A. et al. Case study: Prolonged infectious SARS-CoV-2 Shedding from an asymptomatic immunocompromised individual with cancer. Cell 183, 1901-1912.e9 (2020)

15. Rueca, M. et al. Compartmentalized replication of SARS-Cov-2 in upper vs. lower respiratory tract assessed by whole genome quasispecies analysis. Microorganisms 8, 66 (2020).

16. Ramazzotti, D. et al. VERSO: A comprehensive framework for the inference of robust phylogenies and the quantification of intrahost genomic diversity of viral samples. Patterns 2, 100212 (2021).

17. Jary, A. et al. Evolution of viral quasispecies during SARS-CoV-2 infection. Clin. Microbiol. Infect. https://doi.org/10.1016/j.cmi. 2020.07.032 (2020).

18. Capobianchi, M. R. et al. Molecular characterization of SARS-CoV-2 from the first case of COVID-19 in Italy. Clin. Microbiol. Infect. 26, 954-956 (2020).

19. Armero, A., Berthet, N. \& Avarre, J.-C. Intra-host diversity of SARS-Cov-2 should not be neglected: Case of the state of Victoria, Australia. Viruses 13, 133 (2021).

20. $\mathrm{Xu}$, Y. et al. Dynamics of severe acute respiratory syndrome coronavirus 2 genome variants in the feces during convalescence. J. Genet. Genomics 47, 610-617 (2020).

21. Popa, A. et al. Genomic epidemiology of superspreading events in Austria reveals mutational dynamics and transmission properties of SARS-CoV-2. Sci. Transl. Med. 12, 66 (2020).

22. Lythgoe, K. A. et al. SARS-CoV-2 within-host diversity and transmission. Science https://doi.org/10.1126/science.abg0821 (2021).

23. Pfefferle, S. et al. SARS Coronavirus-2 variant tracing within the first Coronavirus Disease 19 clusters in northern Germany. Clin. Microbiol. Infect. 27(130), e5-130.e8 (2021).

24. James, S. E. et al. High resolution analysis of transmission dynamics of Sars-Cov-2 in two major hospital outbreaks in South Africa leveraging intrahost diversity. medRxiv https://doi.org/10.1101/2020.11.15.20231993 (2020).

25. Shen, Z. et al. Genomic diversity of severe acute respiratory syndrome-coronavirus 2 in patients with coronavirus disease 2019. Clin. Infect. Dis. 71, 713-720 (2020).

26. Wang, D. et al. Population bottlenecks and intra-host evolution during human-to-human transmission of SARS-CoV-2. Front. Med. 8, 66 (2021).

27. Goyal, A., Reeves, D. \& Schiffer, J. T. Early super-spreader events are a likely determinant of novel SARS-CoV-2 variant predominance. medRxiv https://doi.org/10.1101/2021.03.23.21254185 (2021).

28. Wölfel, R. et al. Virological assessment of hospitalized patients with COVID-2019. Nature 581, 465-469 (2020).

29. Focosi, D. \& Maggi, F. Neutralising antibody escape of SARS-CoV-2 spike protein: Risk assessment for antibody-based Covid-19 therapeutics and vaccines. Rev. Med. Virol. https://doi.org/10.1002/rmv.2231 (2021).

30. Grint, D. J. et al. Case fatality risk of the SARS-CoV-2 variant of concern B.1.1.7 in England, 16 November to 5 February. Euro Surveill 26, 66 (2021).

31. Grabowski, F., Preibisch, G., Giziński, S., Kochańczyk, M. \& Lipniacki, T. SARS-CoV-2 variant of concern 202012/01 has about twofold replicative advantage and acquires concerning mutations. Viruses 13, 66 (2021).

32. Cele, S. et al. Escape of SARS-CoV-2 501Y.V2 from neutralization by convalescent plasma. Nature https://doi.org/10.1038/s41586021-03471-w (2021).

33. Wang, P. et al. Antibody resistance of SARS-CoV-2 variants B.1.351 and B.1.1.7.. bioRxiv https://doi.org/10.1101/2021.01.25.428137 (2021).

34. Widera, M. et al. Bamlanivimab does not neutralize two SARS-CoV-2 variants carrying E484K in vitro. medRxiv https://doi.org/ $10.1101 / 2021.02 .24 .21252372(2021)$.

35. Shen, X. et al. Neutralization of SARS-CoV-2 variants B.1.429 and B.1.351. N. Engl. J. Med. 6, 66 (2021).

36. Garcia-Beltran, W. F. et al. Multiple SARS-CoV-2 variants escape neutralization by vaccine-induced humoral immunity. medRxiv https://doi.org/10.1101/2021.02.14.21251704 (2021).

37. Madhi, S. A. et al. Efficacy of the ChAdOx1 nCoV-19 Covid-19 vaccine against the B.1.351 variant. N. Engl. J. Med. 6, 66 (2021).

38. Kissler, S. M. et al. Densely sampled viral trajectories suggest longer duration of acute infection with B.1.1.7 variant relative to non-B.1.1.7 SARS-CoV-2. medRxiv https://doi.org/10.1101/2021.02.16.21251535 (2021).

39. Domingo, E. \& Perales, C. Viral quasispecies. PLoS Genet 15, e1008271 (2019).

40. CDC. COVID-19 Quarantine and Isolation. Centers for Disease Control and Prevention https://www.cdc.gov/coronavirus/2019ncov/your-health/quarantine-isolation.html (2021)

41. CDC. Ending Isolation and Precautions for People with COVID-19: Interim Guidance. Centers for Disease Control and Prevention https://www.cdc.gov/coronavirus/2019-ncov/hcp/duration-isolation.html (2021).

42. Marks, M. et al. Transmission of COVID-19 in 282 clusters in Catalonia, Spain: A cohort study. Lancet Infect. Dis. 6, 66 (2021). 
43. CDC. COVID-19 and Your Health. Centers for Disease Control and Prevention https://www.cdc.gov/coronavirus/2019-ncov/ifyou-are-sick/end-home-isolation.html (2020).

44. van Kampen, J. J. A. et al. Duration and key determinants of infectious virus shedding in hospitalized patients with coronavirus disease-2019 (COVID-19). Nat. Commun. 12, 267 (2021).

45. La Scola, B. et al. Viral RNA load as determined by cell culture as a management tool for discharge of SARS-CoV-2 patients from infectious disease wards. Eur. J. Clin. Microbiol. Infect. Dis. https://doi.org/10.1007/s10096-020-03913-9 (2020).

46. Rhee, C., Kanjilal, S., Baker, M. \& Klompas, M. Duration of severe acute respiratory syndrome coronavirus 2 (SARS-CoV-2) infectivity: When is it safe to discontinue isolation?. Clin. Infect. Dis. https://doi.org/10.1093/cid/ciaa1249 (2020).

47. Rodríguez-Grande, C. et al. Inference of active viral replication in cases with sustained positive reverse transcription-PCR results for SARS-CoV-2. J. Clin. Microbiol. 59, 66 (2021).

48. Dimcheff, D. E. et al. SARS-CoV-2 total and subgenomic RNA viral load in hospitalized patients. medRxiv https://doi.org/10.1101/ 2021.02.25.21252493 (2021).

49. Lavine, J. S., Bjornstad, O. N. \& Antia, R. Immunological characteristics govern the transition of COVID-19 to endemicity. Science 371, 741-745 (2021).

50. Herbeck, J. T. et al. Is the virulence of HIV changing? A meta-analysis of trends in prognostic markers of HIV disease progression and transmission. AIDS 26, 193-205 (2012).

51. Watanabe, T. \& Kawaoka, Y. Pathogenesis of the 1918 pandemic influenza virus. PLoS Pathog. 7, 66 (2011).

52. Marshall, I. D. \& Fenner, F. Studies in the epidemiology of infectious myxomatosis of rabbits. V. Changes in the innate resistance of Australian wild rabbits exposed to myxomatosis. J. Hyg. (Lond.) 56, 288-302 (1958).

53. Kerr, P. J. et al. Next step in the ongoing arms race between myxoma virus and wild rabbits in Australia is a novel disease phenotype. PNAS https://doi.org/10.1073/pnas.1710336114 (2017).

54. Best, S. M. \& Kerr, P. J. Coevolution of host and virus: the pathogenesis of virulent and attenuated strains of myxoma virus in resistant and susceptible European rabbits. Virology 267, 36-48 (2000).

55. Karlin, S. A First Course in Stochastic Processes (Academic Press, 1966).

56. Li, Q. et al. Prolonged shedding of severe acute respiratory syndrome coronavirus 2 in patients with COVID-19. Emerg. Microb. Infect. 9, 2571-2577 (2020)

57. Bar-On, Y. M., Flamholz, A., Phillips, R. \& Milo, R. SARS-CoV-2 (COVID-19) by the numbers. eLife 9, 66 (2020).

\title{
Author contributions
}

D.V.E., M.S., and A.C. conceived the original study design. D.V.E. and A.C. designed the mathematical modeling approach and wrote the first draft. D.V.E. conducted the modeling, with input from M.S. and A.C. A.N. and U.T. contributed text to the Introduction, Discussion and Supplemental Materials. All authors edited and provided input into the manuscript. D.J.-M. and A.C. supervised the study, with input and feedback on specific topics from B.Z. and M.S.R.

\section{Funding}

A.N. acknowledges funding from the National Science Foundation Graduate Research Fellowship Program under Grant No. DGE-1762114. Any opinions, findings, and conclusions or recommendations expressed in this material are those of the author(s) and do not necessarily reflect the views of the National Science Foundation. Fractal Therapeutics provided support in the form of salaries for authors M.S, and U.T., but did not have any additional role in the study design, data collection and analysis, decision to publish, or preparation of the manuscript. The specific roles of these authors are articulated in the 'author contributions' section.

\section{Competing interests}

A.C., M.S., and U.T. are employees and shareholders of Fractal Therapeutics. D.V.E., A.N., and D.J.-M. are shareholders of Fractal Therapeutics. This does not alter our adherence to journal policies on sharing data and materials. B.Z. and M.R. do not declare any competing interests.

\section{Additional information}

Supplementary Information The online version contains supplementary material available at https://doi.org/ 10.1038/s41598-021-02148-8.

Correspondence and requests for materials should be addressed to A.C.

Reprints and permissions information is available at www.nature.com/reprints.

Publisher's note Springer Nature remains neutral with regard to jurisdictional claims in published maps and institutional affiliations.

\begin{abstract}
Open Access This article is licensed under a Creative Commons Attribution 4.0 International License, which permits use, sharing, adaptation, distribution and reproduction in any medium or format, as long as you give appropriate credit to the original author(s) and the source, provide a link to the Creative Commons licence, and indicate if changes were made. The images or other third party material in this article are included in the article's Creative Commons licence, unless indicated otherwise in a credit line to the material. If material is not included in the article's Creative Commons licence and your intended use is not permitted by statutory regulation or exceeds the permitted use, you will need to obtain permission directly from the copyright holder. To view a copy of this licence, visit http://creativecommons.org/licenses/by/4.0/.
\end{abstract}

(c) The Author(s) 2021 